pressure 15 hours after work was $120.3 \quad(11.7) / 77.0 \quad(7.5)$ [mmHg]; mean FeNO 19.0 ppb (7.6). Mean blood levels of C reactive protein and IL- 8 were $2.5 \mu \mathrm{g} / \mathrm{ml}(3.7)$ and $12.2 \mathrm{pg} / \mathrm{ml}$ (3.8) respectively.

Conclusions Our database serves as a basis to investigate shortterm health effects using mixed effect regression models. We hypothesise to find particle related changes in heart rate variability and inflammation markers and we will investigate combined health effects of particles and noise. The variable exposure and the low association between particles and noise are a good opportunity to study health outcomes related to these two exposure types in the near-road environment.

This abstract does not necessarily represent US EPA policy.

\section{EXPOSURE ASSESSMENT FOR A CANADIAN CENSUS COHORT STUDY OF NIGHT SHIFT WORK AND CANCER RISKS}

'M A Harris, ${ }^{2}$ Demers. 'Ryerson University, Toronto, Canada; ${ }^{2}$ Occupational Cancer Research Centre, Cancer Care Ontario, Toronto, Canada

\subsection{6/oemed-2013-101717.388}

Objectives Shift work has been categorised as a probable human carcinogen by the International Agency for Research on Cancer. This is an exposure assessment for a study of shift work and cancer in a cohort created by linkage of the Canadian national cancer registry and the 1991 long form census, which recorded occupation and industry of employment for 2.1 million Canadians, but did not query specific exposures. We used a contemporary (1993) survey to characterise shift work exposures by occupation, industry, and sex.

Methods Analyses were conducted on the 1993 Survey of Labour and Income Dynamics (SLID) to determine the prevalence of night shift work in the contemporary population, highly exposed industries and occupations and sex differences in shift work prevalence within industries. All analyses were restricted to the employed population and weighted to account for sampling methodology.

Results 17\% of employed 1993 SLID respondents were exposed to night shift work, with $5 \%$ of reporting a regular evening work schedule, $2 \%$ a regular night shift and $10 \%$ a rotating shift. Night shift work was most common $(>65 \%)$ in pulping control operators in the pulp and paper industry; food service helpers, servers and bartenders in hotels and motels; uncommissioned police officers; and light duty cleaners. Exposure to shift work was similar in men and women (18\% vs $16 \%)$, but sex differences were apparent in certain industries. Within protective services (includes police) shift work prevalence was $11 \%$ in women and $20 \%$ in men while within hospitals prevalence was $37 \%$ in women and $29 \%$ in men.

Conclusions This exposure assessment for a census cohort has the advantage of drawing from a contemporary population based sample, demonstrating that occupation, industry and sex are important dimensions for a shift work exposure matrix designed for application to a census cohort or other general population sample.

\section{Session: 32. Agriculture and cancer}

\section{RISK OF TOTAL AND AGGRESSIVE PROSTATE CANCER AND PESTICIDE USE IN THE AGRICULTURAL HEALTH} STUDY
SK Koutros. National Cancer Institute, Rockville, United Stated of America

\subsection{6/oemed-2013-101717.389}

Objectives Pesticides have been associated with prostate cancer risk, but few studies have evaluated specific pesticides and studies have not explored differences by subtype to identify important risk for the more lethal, aggressive, form of prostate cancer. Therefore, we studied the risk of prostate cancer associated with specific pesticides among 1,962 incident cases, including 919 cases of aggressive prostate cancer (distant Stage or poorly differentiated or Gleason $\geq 7$ or fatal prostate cancer) diagnosed between 1993 and 2007 from 54,412 men of the Agricultural Health Study (AHS) cohort.

Methods Poisson regression analysis was used to calculate rate ratios (RR) and 95\% confidence intervals $(95 \% \mathrm{CI})$ for lifetime use of 48 pesticides and prostate cancer incidence.

Results There was no overall association between any specific pesticide and prostate cancer risk. However, three organophosphate insecticides were significantly associated with aggressive prostate cancer: fonofos (RR for the highest quartile of exposure (Q4) versus nonexposed $=1.63$, 95\% CI: 1.22-2.17; p-trend $<0.001$ ), malathion (RR for Q4 versus nonexposed $=1.43,95 \%$ CI: $1.08-1.88$; p-trend $=0.04)$, and terbufos $(\mathrm{RR}$ for $\mathrm{Q} 4$ versus nonexposed $=1.29,95 \%$ CI: $1.02-1.64$; p-trend $=0.03)$. The organochlorine insecticide aldrin was also significantly associated with risk of aggressive prostate cancer with a RR for Q4 versus nonexposed $=1.49,95 \%$ CI: $1.03-2.18$; -trend $=0.02$.

Conclusions Four insecticides were observed to increase the risk of aggressive prostate cancer in the AHS. Advantages of this analysis over previous analyses include a large number of prostate cancer cases and detailed information on lifetime use of specific pesticides. This is the first time specific pesticides have been studied and implicated as risk factors for aggressive prostate cancer and may suggest that pesticides play a role in prostate cancer progression rather than at the earlier initiation stage of transformation.

\section{THE PIPAH STUDY: A NEW PROSPECTIVE STUDY OF PESTICIDE APPLICATORS}

${ }^{1}$ Ah Harding, ${ }^{1}$ Frost, ${ }^{2}$ Chen, ${ }^{1}$ Boorman, ${ }^{3}$ Pearce, ${ }^{1}$ Fishwick. ${ }^{1}$ Health \& Safety Laboratory, Buxton, United Kingdom; ${ }^{2}$ Health \& Safety Executive, Bootle, United Kingdom; ${ }^{3}$ London School of Hygiene and Tropical Medicine, London, United Kingdom

\subsection{6/oemed-2013-101717.390}

Objectives The PIPAH Study, a new prospective study of professional pesticide applicators in Great Britain is being established. The objectives of the study will be to monitor the long-term health of these pesticide applicators and to investigate associations between health outcomes and occupational exposure to pesticides. Methods The 21,000 members of two national registers of professional pesticide applicators will be invited to participate in the study. The schedule of reminders includes a postcard sent to all potential participants shortly after the initial invitation, an article in the trade journal for pesticide applicators, and a full study pack sent to non-responders. Those who agree to participate will complete a general questionnaire covering their work history, previous pesticide usage, personal and family medical history, signs/symptoms of neurological disease, socioeconomic factors, diet and lifestyle. This can be completed using the paper questionnaire sent to them or online. New members of the two registers will be invited to participate in the study in a rolling recruitment programme. 